OPEN ACCESS

Edited by:

Mingbo Yin,

Fudan University, China

Reviewed by:

Olivier Glaizot,

University of Lausanne, Switzerland

Rita Žiegyte

Nature Research Center, Lithuania Aneliya Borisova Bobeva,

Bulgarian Academy of

Sciences, Bulgaria

*Correspondence:

Rafael Gutiérrez-López rgutierrez@cibio.up.pt; rafgutlop@gmail.com

Specialty section:

This article was submitted to Behavioral and Evolutionary Ecology,

a section of the journal

Frontiers in Ecology and Evolution

Received: 03 June 2020

Accepted: 13 August 2020

Published: 18 September 2020

Citation:

Gutiérrez-López R, Bourret $V$ and Loiseau C (2020) Is Host Selection by Mosquitoes Driving Vector Specificity of Parasites? A Review on the Avian

Malaria Model.

Front. Ecol. Evol. 8:569230

doi: 10.3389/fevo.2020.569230

\section{Is Host Selection by Mosquitoes Driving Vector Specificity of Parasites? A Review on the Avian Malaria Model}

\author{
Rafael Gutiérrez-López ${ }^{1 *}$, Vincent Bourret ${ }^{1}$ and Claire Loiseau ${ }^{1,2}$ \\ ${ }^{1}$ Laboratório Associado, CIBIO, Centro de Investigação em Biodiversidade e Recursos Genéticos, InBio, University of Porto, \\ Campus Agrário de Vairão, Vairão, Portugal, ${ }^{2}$ CEFE, Université de Montpellier, CNRS, Montpellier, France
}

Parasites and hosts are in a complex evolutionary arm race where host compatibility represents a key obstacle for successful infections. The degree of parasite specialization on a host varies along a continuum: on one end, extreme specialist parasites may be restricted to a single host species, and on the other, generalist parasites are able to infect a diverse set of hosts. Multiple intrinsic factors associated to the host, such as their immune system and physiological condition, can contribute to the evolution of host specificity of parasites and have been well-studied and documented in vertebrates. In contrast, vector specificity of parasites has been largely overlooked, especially in natural conditions. While a few studies suggested that insect vectors do not play an important role in shaping the parasite community structure since they may feed widely from the bird community, current studies have demonstrated the importance of vector feeding behavior on transmission dynamics for several vector-borne pathogens. Here, we provide a review on the specialization of avian Plasmodium in vectors, emphasizing the necessity to study simultaneously the three players to further understand how host choice by the vectors might influence the distribution of parasites in the wild. In addition, we suggest specific research directions making use of both empirical data gathered in the field and controlled experiments on vector host-feeding preferences.

Keywords: avian plasmodium, feeding preference, insect-vector, ornithophilic, parasite specialization

\section{INTRODUCTION}

The degree of parasite specialization for a host varies along a continuum: on one end, extreme specialist parasites may be restricted to a single host species, and on the other, generalist parasites are able to infect a diverse set of hosts, with a large diversity of intermediate states (Vázquez et al., 2005; Poulin, 2007; Hellgren et al., 2009; Mccoy et al., 2013). Both ecological and evolutionary mechanisms are currently acting to maintain this high diversity in the range of host specialization (Poisot et al., 2015). Understanding how parasite specialization has evolved and how different strategies can co-occur is challenging and remains a debated topic. It has been suggested first that 
specialization represents an evolutionary "dead end," which limits further evolution (Kelley and Farrell, 1998; Snyder and Loker, 2000; Nosil, 2002). However, several later studies have indicated that generalists can repeatedly evolve from specialist lineages, albeit with a relatively low frequency (Poulin et al., 2006; Johnson et al., 2009; Gomez et al., 2010).

The probability for a parasite of infecting a suitable host depends on many factors, including the host-parasite compatibility, rate of encounter, the parasite life cycle, the population density (Combes, 1997) as well as host intrinsic factors such as its immune system, age, or sex (Loiseau et al., 2008; Bichet et al., 2014; Calero-Riestra and Garcia, 2016). For parasites such as avian Plasmodium, with a complex life cycle that involves two hosts, one vertebrate (bird) and one insect (mosquito; Valkiunas, 2005), additional parameters linked to the insect, such as its feeding behavior or immune system, may also influence parasite specialization (Billingsley and Sinden, 1997; GutiérrezLópez et al., 2020). Interactions with the insects, commonly called vectors (Wilson et al., 2017), are likely important for parasite evolution since mosquitoes are the definitive host, in which sexual reproduction occurs. Thus, as in the vertebrate hosts (hereafter the term "host" will be used), the parasite ingested in a mosquito blood meal must elude the immune system of the vector and complete all the developmental stages while the mosquito remains alive long enough to feed on another host (Marquardt et al., 2005).

Avian malaria parasites are considered an excellent model for investigating parasite transmission dynamics in ecological and evolutionary studies (LaPointe et al., 2012; Rivero and Gandon, 2018). They have been studied extensively in birds because of their high diversity of hosts and nearly worldwide distribution (Valkiunas, 2005). To date, parasite specialization for their vertebrate hosts has been much more studied compared to their vector specificity. Studies have investigated host-parasite associations using phylogenetic analyses and indices of host specialization (e.g., Ishtiaq et al., 2008; Hellgren et al., 2009; Loiseau et al., 2012; Ricklefs et al., 2014; Lauron et al., 2015; Fecchio et al., 2019) and have revealed that several lineages of Plasmodium exhibit extreme host generalist parasitism strategies, whereas other lineages appear to have been constrained to certain host families or individual bird species (Ricklefs and Fallon, 2002; Ricklefs et al., 2004; Beadell et al., 2009). Interestingly, in the discussion of a large number of these publications, authors suggest a key role for vectors in the system, but very few studies are exploring this vector role. In fact, the number of publications using the bird-avian Plasmodium system has considerably increased with on average 25 articles published per year since the last 10 years, but the number of publications that integrates the vector in their study remains much lower (3.5 per year since 2010; see Supplementary Figure 1). A number of reasons are responsible for the fact that vector specificity of avian parasites has been largely overlooked, especially in natural conditions: (i) researchers studying avian malaria are often trained ornithologists with little or no entomological expertise, (ii) insect vectors are difficult to capture compared to birds, (iii) they are also more difficult to identify, especially in tropical countries, (iv) the parasite prevalence in mosquitoes is usually low ( $10 \%$ on average with values ranging from 0.003 to $30 \%$; Aruch et al., 2007; Glaizot et al., 2012; Lalubin et al., 2013; Zélé et al., 2014; Martínez-de la Puente et al., 2015, 2016, 2020) compared to birds where prevalence often reaches up to $80 \%$ (Bichet et al., 2014).

Different ecological and behavioral factors determine the coevolution of avian Plasmodium and mosquitoes (Ferraguti et al., 2016). Among them, the vector feeding behavior is an important parameter that can affect the specialization of parasites both in the vector and the vertebrate host (Figure 1). A hypothesis on parasite vertebrate host specificity is that if the vector is not selective and feeds on a large variety of avian hosts, then the malaria parasite should have an advantage in being an avian host generalist, maintaining compatibility with a wide set of birds, even if some of them are not optimal hosts. This should increase its encounter rate with suitable avian hosts and, therefore, its overall prevalence in the community (Dobson, 2004; Hellgren et al., 2009). On the other hand, if the vector is selective and feeds on specific bird species (Kilpatrick et al., 2006; Hellgren et al., 2008; Rizzoli et al., 2015), the parasite should have an advantage in being host specialist and optimize its host compatibility, development and transmission efficiency. Interestingly, the host specificity of parasites may not reflect their vector specificity. As shown in Figure 1, a parasite that is a vector specialist (i.e., able to complete its sexual cycle in one mosquito species only) could present different host strategies, specialist or generalist, depending on the host selection by vectors. If the parasite is a vector generalist (i.e., able to complete its sexual cycle in various mosquito species), the diversity in the range of host specialization can be even more important i.e., from highly host specialist to extremely host generalist (Figure 1). Therefore, from a parasite perspective, it would seem more advantageous to be a vector generalist since this would enable a broader gradient of host specificity (considering always that those vectors are competent to transmit the parasite). On the other hand, being a vector or host generalist may come at the expense of optimal parasite compatibility with host or vector.

Here, we review the current knowledge on parameters that influence host selection by mosquitoes and its potential repercussions on vector-parasite co-evolution and vector specificity. Using the MalAvi database (Bensch et al., 2009), which currently comprises 1276 unique Plasmodium lineages found in around 1,200 bird species and 40 mosquito species and data from the literature, we aimed at (i) evaluating the feeding preference of those mosquito species that could be potential avian malaria vectors and (ii) exploring the phylogenetic relationships of avian malaria lineages and their vector and bird specificity. We selected mosquito species where Plasmodium lineages have been isolated from the head/thorax or from salivary glands as a proxy of competent vector. We excluded lineages found in whole mosquitoes since they may correspond to parasites that will experience abortive developments in the vector midgut (Valkiunas et al., 2013). Finally, we address various challenges and limitations when working with vectors and suggest lines of research for the future, both in the field and in laboratory conditions. 


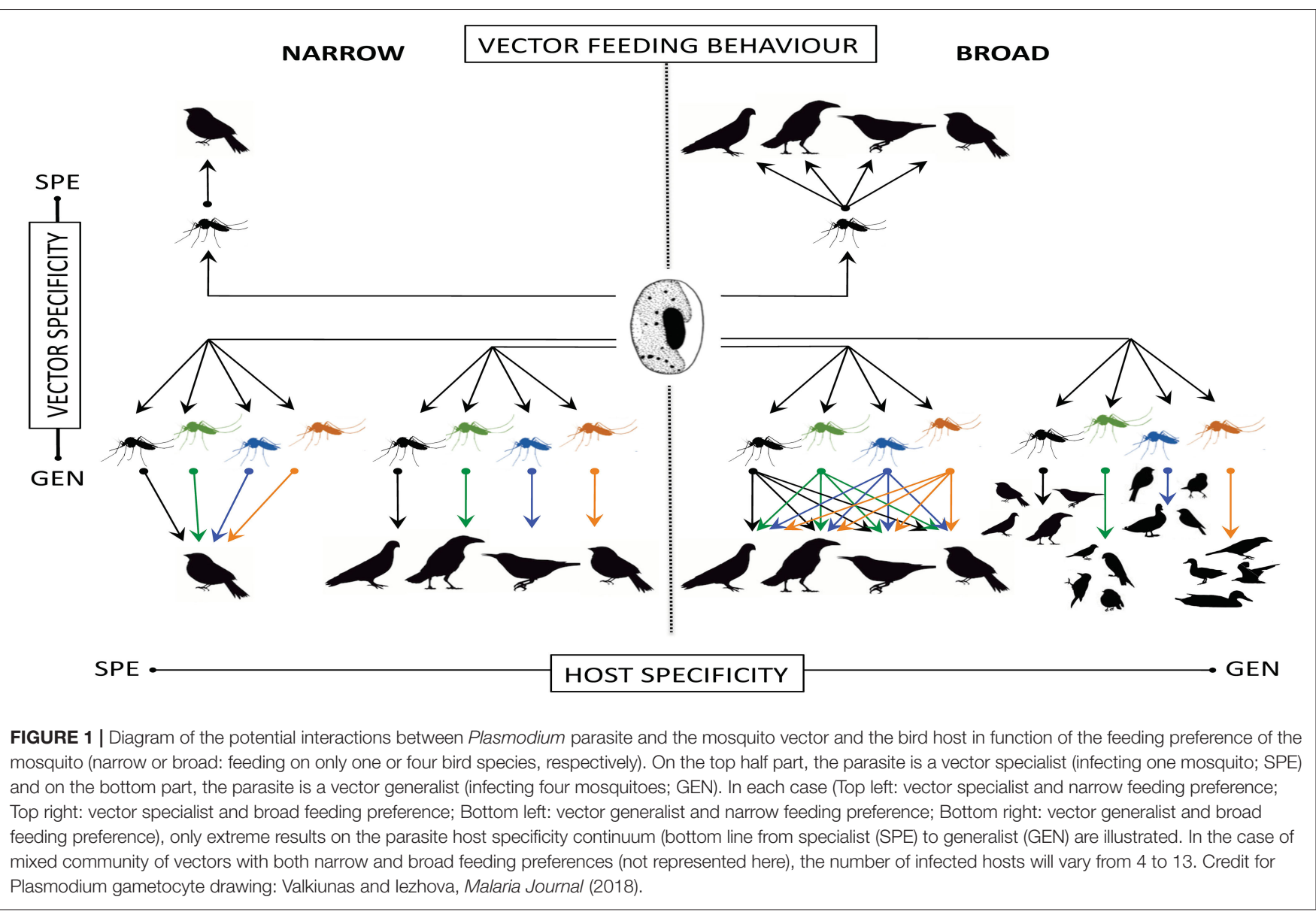

\section{HOW DO MOSQUITOES SELECT THEIR HOSTS?}

\section{Large Variation in Feeding Preferences}

The transmission dynamics of avian Plasmodium is going to be strongly influenced by the contact rates between mosquitoes and susceptible hosts (Takken and Verhulst, 2013). Under natural conditions, mosquitoes show different innate feeding preferences at different levels. First, while some species feed mostly on mammals (i.e., mammophilic species), others prefer to feed on birds (i.e., ornithophilic species), or amphibians and reptiles, while yet other species show a real opportunistic behavior (Molaei et al., 2007; Burkett-Cadena et al., 2008; Viana et al., 2010; Muñoz et al., 2012; Martínez-de la Puente et al., 2015). Second, in addition to this broad tendency for particular host groups, mosquitoes may feed on certain host species at higher rates than expected from their abundance (Hamer et al., 2009; Lura et al., 2012). For instance, in Europe, Cx. pipiens seems to show a preference for blackbirds (Turdus merula) compared to European starlings (Sturnus vulgaris; Rizzoli et al., 2015). These two levels (group/species) of selection by the mosquito could be altered by the availability of preferred hosts that depends on seasonality and habitat (Wekesa et al., 1997; Kilpatrick et al., 2006; Thiemann et al., 2011). As an example,
Culex pipiens and Culex tarsalis, known to be predominantly ornithophilic, shift their diet to include more mammals when the availability of certain bird species decreases at the end of the summer (Kilpatrick et al., 2006). Seasonality in temperate regions affects also mosquito abundance and in consequence parasite prevalence, with a typical peak in August or early autumn (Ferraguti et al., 2013; Lalubin et al., 2013). In addition, more or less effective host anti-mosquito behavior and other intrinsic host factors, developed below, influence feeding patterns of mosquitoes (Takken and Verhulst, 2013).

\section{Host Characteristics Influencing Feeding Preferences}

Female mosquitoes detect their vertebrate hosts by a combination of different cues [e.g., visual cues (body size), carbon dioxide $\left(\mathrm{CO}_{2}\right)$, temperature, moisture, and/or body odor], which influence the attractiveness of individuals to vectors (Eiras and Jepson, 1994; Lehane, 2005). Some individuals (males or females) depending on their physiological state (e.g., hormone levels), age-class groups (young or older individuals) or infectious status (infected vs. non-infected) may be more attractive than others. Those individuals are therefore more likely to receive more mosquito bites (Dye and Hasibeder, 1986; Liebman 
et al., 2014) and acquire new infections from vectors, but also to pass on parasites to subsequent vectors with whom they come in contact. These super-receivers and super-spreaders increase the contact rates between hosts and vectors, and may influence the vector specificity of parasites. Both empirical studies and theoretical models (Woolhouse et al., 1997; Perkins et al., 2013) showed that inter-individual heterogeneity has considerable implications for the epidemiology of mosquitoborne parasites.

First, larger hosts may receive more mosquito bites (Yan et al., 2017), probably due to (i) the higher amounts of cues (e.g., $\mathrm{CO}_{2}$ ) released by larger individuals or (ii) the greater skin surface available for mosquito bite (Kleiber, 1947; Yan et al., 2017). Different studies have reported a positive relationship between the host body mass and the feeding rate of different blood-searching insect vector (Martínezde la Puente et al., 2010; Schönenberger et al., 2016). In addition, Yan et al. (2017) found that Cx. pipiens fed more frequently on birds with a longer tarsus, suggesting that larger areas of exposed skin are important in determining feeding patterns. However, experimental studies have observed contrasting results. While Simpson et al. (2009) found differences in $C x$. pipiens attraction among bird species, with American robins (Turdus migratorius) being more attractive than house sparrows (Passer domesticus), Gutiérrez-López et al. (2019) found no difference in attraction between jackdaws (Coloeus monedula) and house sparrows. Furthermore, in both studies, differences in body mass between individuals of the same species did not determine mosquito attraction, suggesting that, at short distances (as happens in an experimental setup), other signals such as heat, humidity or odor could be more important than intraspecific differences in body mass (Raji and DeGennaro, 2017).

Sex-associated characteristics could also cause differences in the mosquito attraction (Zuk et al., 1990). Sex-biased individual attraction could explain why several studies found that male birds present usually a higher prevalence of blood parasites than females (Skorping and Jensen, 2004; Zuk and Stoehr, 2010; Calero-Riestra and Garcia, 2016). As an example, Burkett-Cadena et al. (2014) found that mosquitoes had a higher feeding preference for male birds, but this result was observed mainly in mammophilic mosquitoes. On the other hand, several studies failed to find any significant sex-biased feeding pattern in ornithophilic mosquitoes (Simpson et al., 2009; Yan et al., 2018; Cozzarolo et al., 2019; Díez-Fernández et al., 2019; Gutiérrez-López et al., 2019). It has also been suggested that the composition of volatile substances from the uropygial gland secretions, which differ between male and female birds (Jacob et al., 1979; Amo et al., 2012), could affect the attraction of blood-sucking insects (Russell and Hunter, 2005; Martínez-de la Puente et al., 2011). However, DíezFernández et al. (2020) found no difference between feeding pattern in Culex pipiens and the sex of the birds. Therefore, other factors such as the degradation of the uropygial secretions by the microbiome present in the feathers could act as an attractive to mosquitoes (Allan et al., 2006; Díez-Fernández et al., 2020).

\section{Parasite Manipulation and Vector Adaptive Avoidance}

According to the parasite manipulation hypothesis (Poulin, 2000), the infection with the parasite should increase the incidence of feeding by mosquitoes (Cornet et al., 2013), either by modifying its defense behavior or increasing its attractiveness (Heil, 2016). It could also modify the behavior of the mosquito, making it more aggressive. These parasite manipulations should increase the contact rates between the host and the vector and, therefore, favor the parasite transmission (Hurd, 2003; Lefévre and Thomas, 2008).

To date, experimental tests of this hypothesis using the Plasmodium relictum-Cx. pipiens system have obtained contradictory results. While some studies found that mosquitoes were attracted to, or fed on chronically infected birds at a higher rate than uninfected hosts (Cornet et al., 2013; DíezFernández et al., 2020), other studies have reported the opposite pattern (Lalubin et al., 2012) or an absence of significant differences between infected and uninfected birds (Yan et al., 2018; Gutiérrez-López et al., 2019). This discrepancy may come from the different experimental procedures used (e.g., either dual-choice olfactometer or direct contact with immobilized hosts) and therefore further studies are required to clarify how the infection status may influence the feeding behavior of mosquitoes.

The parasite load in the vertebrate (i.e., parasitemia) may also play an important role on the mosquito feeding patterns. According to the vector adaptive avoidance (Lalubin et al., 2012), mosquitoes should avoid highly infected individuals due to the costs induced by parasite in the vectors. However, Yan et al. (2018) found the opposite with mosquitoes feeding with a higher rate on those individuals that had a higher Plasmodium parasite load, suggesting a pre-eminence of parasite manipulation over vector avoidance. Comprehensive studies controlling for multiple factors are needed to better understand the interplay between these two opposite factors in the host selection by vectors.

\section{WHAT ARE THE FEEDING PREFERENCES OF MOSQUITOES TRANSMITTING AVIAN MALARIA?}

To illustrate the diversity of mosquito feeding preference patterns, we used the MalAvi database and a set of publications to gather information on blood meals for all the vectors collected in the field with avian Plasmodium in their head/thorax or salivary glands $\left(\mathrm{N}_{\text {vector }}=22\right.$ and $\mathrm{N}_{\text {lineage }}=58$; Figure 2 and see Supplementary Materials, Table S1). We classified the diet, using the percentage of blood meals derived from birds, in three categories as (i) mammophilic (from 0 to 33\%), (ii) opportunistic (from 33 to 66\%), or (iii) ornithophilic (more than 66\%).

According to the literature, $C x$. pipiens was the most studied mosquito species for avian malaria and was found to feed on more than 74 different bird species. In fact, $82 \%$ of the blood meals found in Cx. pipiens were from birds, confirming its ornithophilic feeding preference (Kilpatrick et al., 2006; 

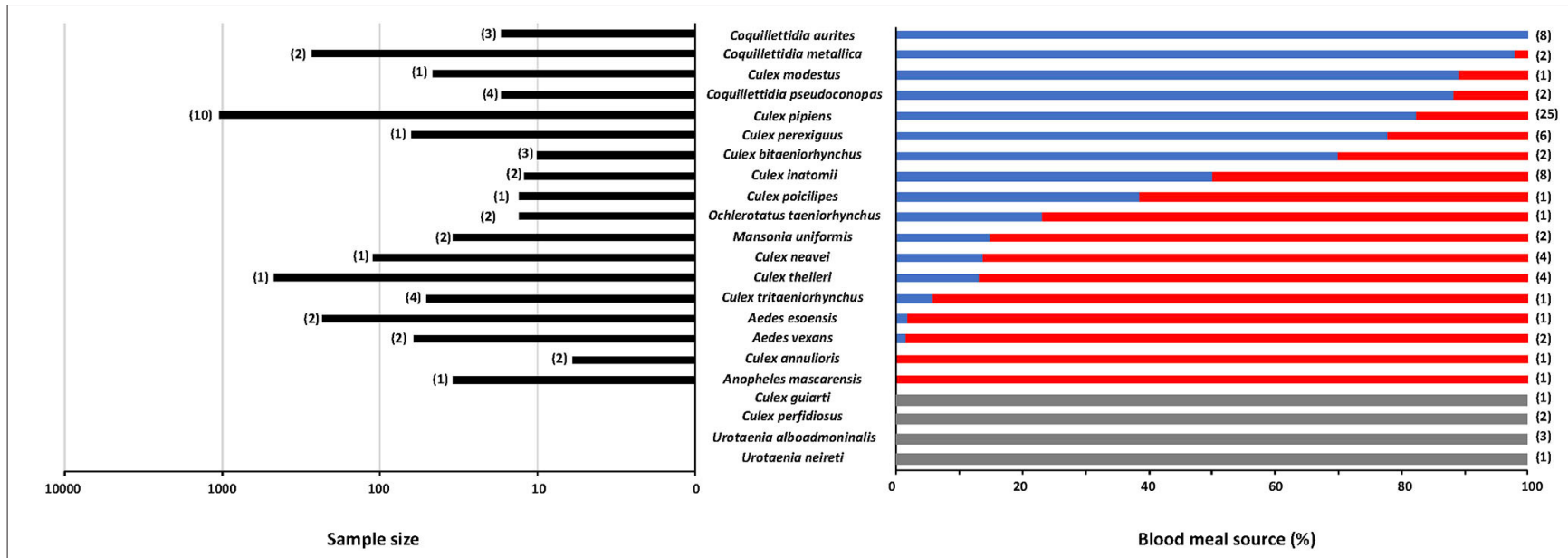

FIGURE 2 | Percentage of bird (blue) and mammal (red) derived blood meals from female mosquitoes species that have shown avian malaria parasites. Mosquito species without information about their blood-feeding behavior are represented in gray $(N=4)$. Numbers in bracket on the right represent the number of different lineages isolated in the head-thorax of each mosquito species. Sample size (log-transformed) of each mosquito species analyzed is shown in black. Numbers in brackets on the left represent the number of studies (see Supplementary Table 1) that have analyzed blood meals in each mosquito species.

Hamer et al., 2008; Figure 2). It also harbors the highest number of avian Plasmodium lineages in the head/thorax $(N=$ 25; Supplementary Figure 2). Therefore, its feeding preference together with its wide distribution around the world, its ecology (Farajollahi et al., 2011) and the diversity of lineages harbored, make this mosquito species one of the main vectors of avian Plasmodium. Among other vector species known to harbor avian Plasmodium, we found species that exhibit mammophilic ( $N$ $=9$; e.g., Culex theileri and Culex neavei), opportunistic $(N=$ 2; Cx. inatomii, Cx. poicilipes), and ornithophilic preferences $(N=7$; Figure 2$)$. We therefore observe a large variation in mosquito behavior that should lead to different vector specificity strategies and parasite transmission efficiency. For instance, avian Plasmodium lineages should enter in contact at higher rate with ornithophilic mosquito species, which preferentially feed on birds, than with opportunistic or mammophilic mosquito species. This in turn should cause avian Plasmodium lineages to become better adapted to ornithophilic mosquitoes and develop specificity for this vector type.

\section{WHAT DO WE KNOW ABOUT VECTOR SPECIFICITY OF AVIAN PLASMODIUM?}

Ten years ago, some studies began to use molecular markers and phylogenetic analyses to examine the distribution of avian blood parasite lineages in wild-caught arthropod vectors. One of the first studies showed that different parasite lineages found in different mosquito genera were sharing a common vertebrate host (Gager et al., 2008). Their data imply that the co-occurrence of two parasite lineages in the same vertebrate host is not necessarily explained by access being provided by a shared mosquito. Other studies showed that different mosquito species could harbor and share closely related or identical parasite lineages (Ishtiaq et al., 2008; Kimura et al.,
2010; Inci et al., 2012), suggesting that these lineages did not have high vector specificity. However, these studies identified lineages from whole mosquitoes or head-thorax and discussed the fact that without salivary gland dissection or experimental demonstration of the transmission cycle, it was not possible to establish the vector competence of mosquitoes for parasite lineages and therefore to fully assess the vector specificity. Indeed, an important percentage of parasite DNA amplification could come from abortive stages of parasite and not from infective stages (i.e., sporozoites; Valkiunas, 2011; Valkiunas et al., 2013). More recently, researchers performed experimental work to identify competent vector species for different avian Plasmodium lineages by studying sporogonic development in salivary glands or saliva (Valkiunas, 2005; Kazlauskiene et al., 2013; Palinauskas et al., 2016; Carlson et al., 2018; Gutiérrez-López et al., 2020). These studies highlighted variation in the mosquito ability to transmit different avian Plasmodium lineages, with some mosquito species being completely refractory to some parasite lineages or some being able to transmit different parasite lineages and/or species.

These experimental results combined with empirical data from field surveys demonstrate that avian Plasmodium lineages, which show extensive variation in host range (from one to more than 100 bird species) and specialization (host specificity index calculated as in Hellgren et al., 2009; Figure 3), also show variation in vector specificity (from one to 6 mosquito species; Figure 3). It is worth noting that the most host generalist parasite (Plasmodium relictum SGS1) that has been found to infect around 120 bird species is not the lineage that has been found in the most vector species so far (Figure 3). Some host specialist lineages (e.g., CXPIP10) or with an intermediate host range (e.g., PADOM02) were found in the same number of vectors as Plasmodium SGS1 (Figure 3), reflecting differential host and vector specificity for a given parasite lineage. Overall however, the parasite host specificity was positively correlated to 


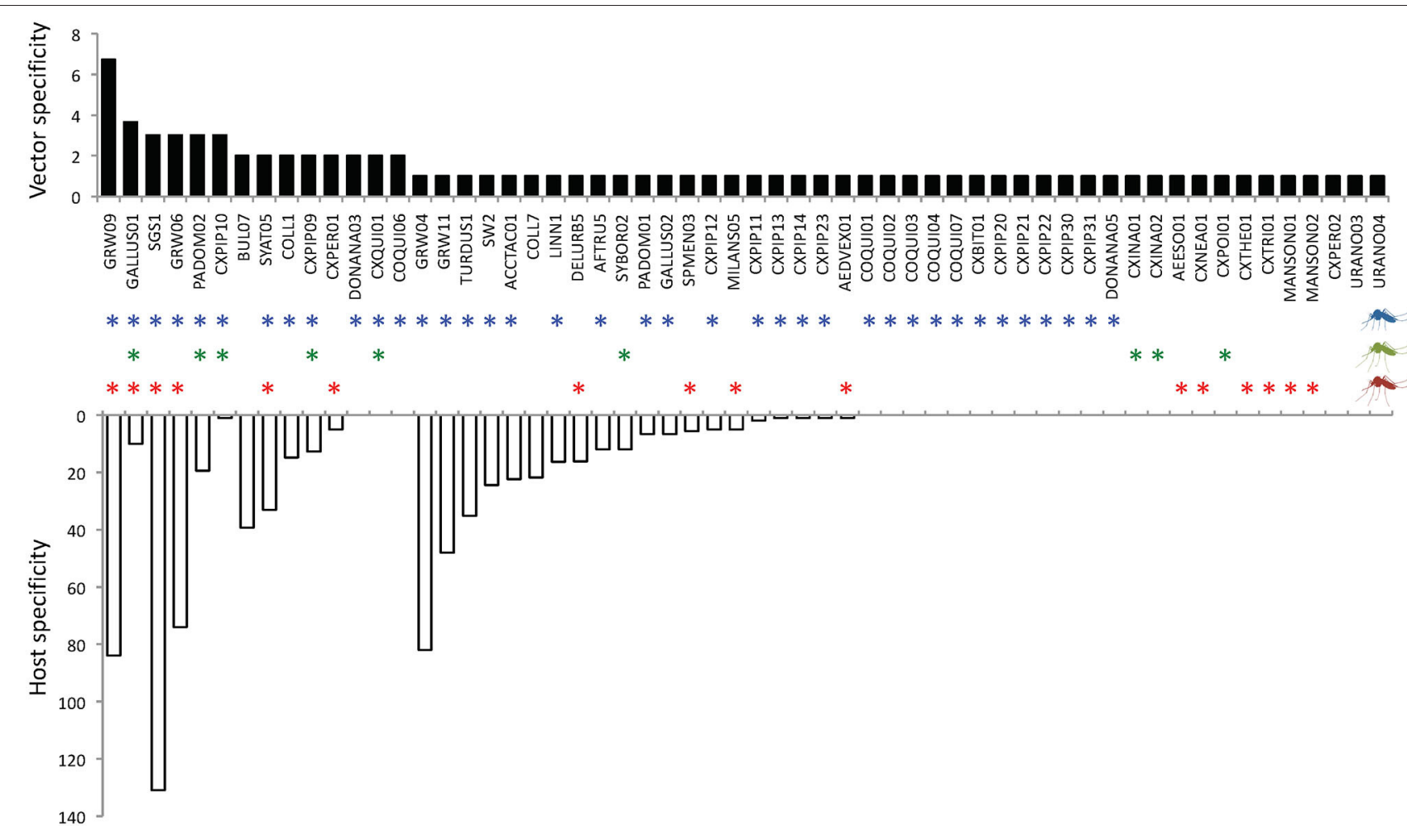

FIGURE 3 | Vector specificity of parasites of the 58 Plasmodium lineages found in head and thorax of vectors and the associated host specificity of lineages that were found in birds $(N=31)$. Blue, green, and red asterisks correspond to lineages that were found in ornithophilic, opportunistic and mammophilic mosquitoes, respectively.

its vector specificity (Pearson coefficient $\mathrm{R}=0.47 ; P=0.008$; see Supplementary Figure 3).

In order to explore the phylogenetic relationships of Plasmodium lineages and their associations with mosquito species, we performed Bayesian phylogenetic analyses with 58 Plasmodium lineages plus another 16 morphospecies described and deposited in MalAvi (Figure 4; see methods in Supplemental Materials). In the tree, we were able to determine 12 clades that correspond to groups of lineages closely related (Clades A to L; represented by a triangular tip with a node support higher than 0.85), most of them including a known morphospecies. First, we observe that most lineages found in the same mosquito species were not necessarily closely related but rather were found all across the phylogenetic tree, suggesting no evidence for strong coevolutionary relationships between these lineages and their vectors. At the lineage level, it appears that around $75 \%$ of the lineages were found in only one vector species, which probably does not always reflect strict vector specificity but rather a sampling effort bias. The other lineages were found in two to six different mosquito species sometimes from different genus (e.g., Plasmodium elongatum GRW06, Plasmodium gallinaceum GALLUS1, Plasmodium GRW09). These three lineages are most likely vector generalists since they shared different mosquito genus that also had different feeding preferences (Figure 4). Interestingly, at the clade level, we found more variation in vector specificity. If we consider a gradient of specialization, from generalist to specialist, we could discern: (i) clades associated with two or three mosquito genera (2-6 species; clades B, D, E, H, J, and L) with different feeding preferences (i.e., vector generalists), (ii) clades associated with only one genus (Culex) but 2-4 different species with different feeding preferences as well (i.e., intermediate vector specificity; clades C, F, and I), and (iii) clades associated with only one or two ornithophilic mosquito species (i.e., vector specialists; Clades A, G, and $\mathrm{K})$.

\section{DOES HOST SELECTION BY THE MOSQUITO INFLUENCE PARASITE VECTOR SPECIFICITY?}

Depending on the feeding preference of the vectors, parasites should have different strategies of transmission and specificity. We predicted that avian Plasmodium lineages found in ornithophilic vectors should be more numerous and on average more vector specialists than lineages found in opportunistic or mammophilic mosquitoes since they have a higher chance of ending up in avian hosts. First, we found that ornithophilic mosquitoes harbor more Plasmodium lineages $(N=38)$ than opportunistic or mammophilic vectors as predicted (9 and 16 lineages, respectively; Figure 3). In addition, all the clades were associated with at least one ornithophilic mosquito, which 


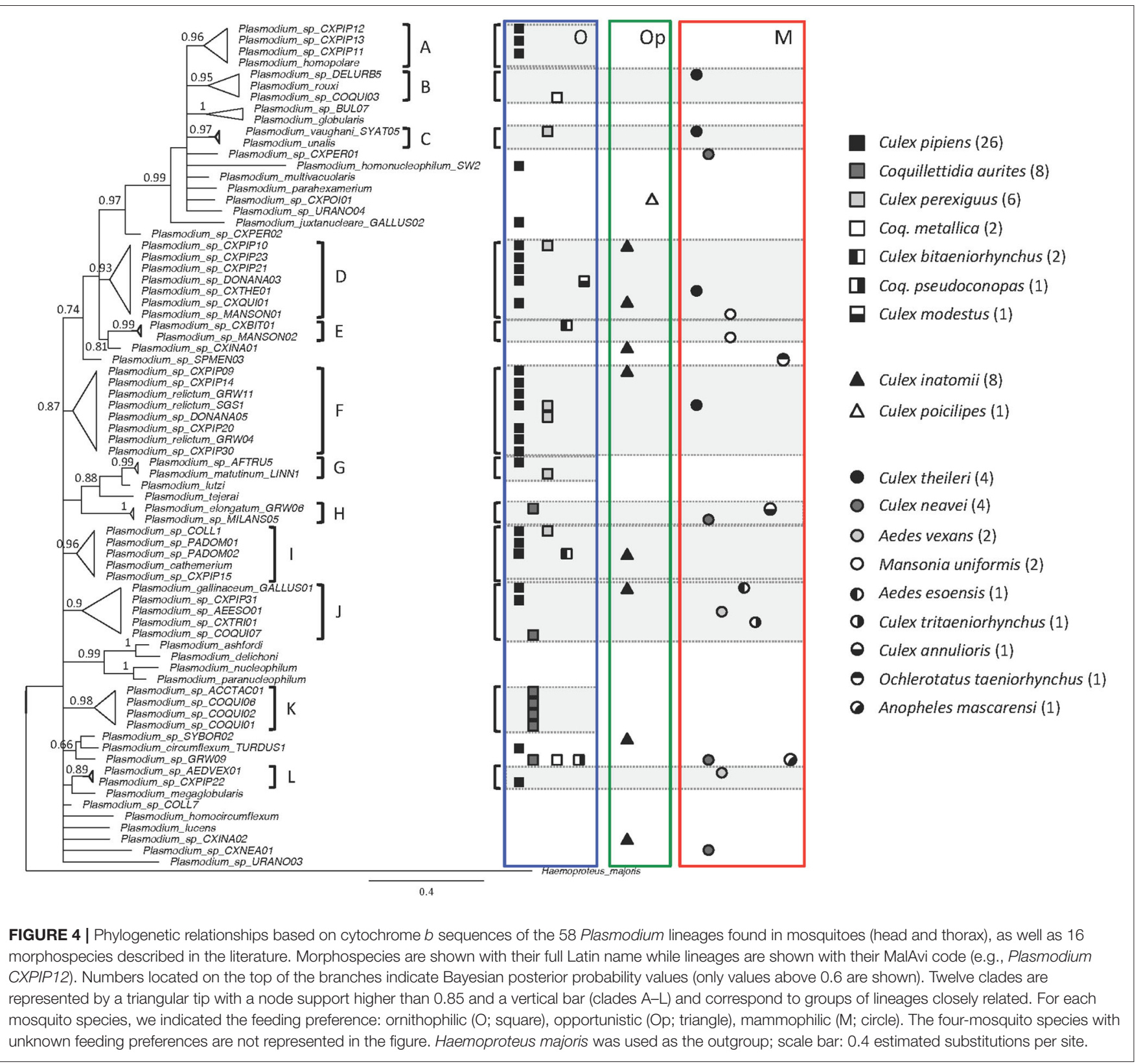

was not the case for opportunistic or mammophilic species. However, the feeding preference of each mosquito species (i.e., the percentage of blood meal from birds, ranging from 0 to $100 \%$; Figure 2) was not significantly associated with the number of Plasmodium lineages that they carried (Pearson coefficient $\mathrm{R}=0.39 ; P=0.11$; see Supplementary Figure 4). We also failed to find statistical associations between the vector specificity index and feeding preference, either at the lineage level $\left(\mathrm{F}_{2,62}=0.64 ; P=0.53\right)$ or clade level $\left(\mathrm{F}_{2,22}=1.83 ; P\right.$ $=0.18$; see Supplementary Figure 5). However, this could be due to insufficient data being available from scarce studies on mosquito-parasite interactions. Most of the lineages are probably transmitted by different mosquito species and therefore the vector specificity of Plasmodium lineages calculated here does not reflect the true parasite specialization. Therefore, it seems difficult to have a clear answer to our question at present since we cannot decipher if host selection is driving or not the vector specificity of avian Plasmodium. Nonetheless, some of these limitations can be addressed by doing extensive mosquito surveys and experimental work in the field that could fill the current knowledge gaps.

\section{HOW CAN WE DETERMINE THE VECTOR SPECIFICITY OF AVIAN PLASMODIUM? CHALLENGES AND FUTURE RESEARCH}

Experimental studies have provided essential information about the vector competence of different mosquito species for 
avian Plasmodium (Santiago-Alarcon et al., 2012; Palinauskas et al., 2016; Gutiérrez-López et al., 2020). Nonetheless, it also seems essential to carry out more holistic studies in natural conditions to allow a better understanding of all the potential interactions between avian Plasmodium, their vectors and their vertebrate hosts. Today, molecular techniques greatly facilitate the identification of blood meal origin in mosquitoes (Alcaide et al., 2009), as well as the presence of blood parasites in different parts of the mosquito body (Njabo et al., 2011; GutiérrezLópez et al., 2016; Palinauskas et al., 2016). However, the number of studies combining both the feeding behavior of mosquitoes and the transmission of avian Plasmodium are still very rare (Ejiri et al., 2011; Ferraguti et al., 2013; Martínezde la Puente et al., 2016), mainly due to the difficult task of capturing engorged mosquitoes during short field seasons. Other vector-parasite systems suffer from the same fieldwork limitations, and to date, very few studies investigated the vector specificity in closely related haemosporidian parasites (Leucocytozoon: Hellgren et al., 2008; Haemoproteus: Martínezde la Puente et al., 2011) or other blood parasites (e.g., Trypanosoma: Bennett, 1961; Svobodová et al., 2017). More efforts should be put on regular and constant surveys using various resting boxes to increase resting insects' collections and blood fed females (Panella et al., 2011) even though it is particularly time consuming. One unusual but appealing solution to overcome this field work limitation and to perform more efficient research is to foster multidisciplinary collaborations between research teams and centers for diseases control to (i) mutualize material and human resources, and (ii) answer different questions using the same specimens. More collaborative work would avoid double laborious field surveys while greatly enhancing the results since one mosquito can be used by different researchers and be screened for a great variety of parasites. For example, the feeding behavior of mosquitoes has been extensively studied in North America. However, due to the spread of West Nile Virus [CDC (Centers for Disease Control and Prevention) (2019)], studies mainly focused on arboviruses (Savage et al., 2007; Farajollahi et al., 2011) while to our knowledge very few studies have investigated avian malaria in vectors (Kimura et al., 2010; Carlson et al., 2018). Something similar can be observed in Central Africa for example, where the main entomological studies have been based on anthropophilic mosquitoes and human malaria or other zoonotic diseases of medical importance (Kamgang et al., 2012; Mayi et al., 2020), while the role of mosquito species in the transmission of avian Plasmodium is practically unknown (Njabo et al., 2009, 2011). If researchers with different research interests but working in the same region or localities were willing to combine their sampling effort, material and data, it would be beneficial for everyone and likely add value to everybody's data. One obvious advantage would be to determine natural co-infection rates in mosquito species and their consequences on pathogen transmission. Although this idea may seem utopian in the ever more specialized research world, it would be a very interesting prospect to greatly increase mosquitoes sampling size from field surveys while exploring mosquito-avian parasite interactions in more details.
To date, $C x$. pipiens has been considered as the main vector of avian malaria in temperate regions. This mosquito species has a wide geographical distribution and is certainly the easiest ornithophilic species to catch and raise in laboratory, which makes it a perfect study model for experimental work. Studies have shown so far that 12 morphospecies of avian Plasmodium are able to develop sporozoites in Cx. pipiens (Santiago-Alarcon et al., 2012; Palinauskas et al., 2016; Gutiérrez-López et al., 2020). However, other less abundant species that share habitats with $C x$. pipiens, such as $C x$. perexiguus, $C x$. restuans, or $C x$. inatomii, among others, may play an important secondary role as vectors of avian malaria parasites. Further research work should therefore focus on less studied mosquitoes that present different feeding behaviors or restricted geographical distribution compared to $C x$. pipiens, although some other potential vectors may be difficult to raise and study. For example, opportunistic mosquitoes might feed on a smaller number of bird species, and thus, come into contact with a smaller number of avian Plasmodium parasites, leading to different co-evolutionary processes between mosquito and avian Plasmodium. In addition, according to the MalAvi database, studies that have identified Plasmodium lineages in insects have been carried out in 20 countries only, mostly in Europe. Thus, future research projects could be conducted in geographical areas that are poorly studied up until now, such as tropical regions and migratory bird wintering areas in Africa, that may present very distinct host selection patterns and vector specificity due to the highly diverse communities of hosts and vectors. Undoubtedly, the development of field surveys and experimental studies on vector competence of poorly sampled or little-known mosquito species is essential to better understand the vector specificity and transmission dynamics of avian Plasmodium.

Lastly, detection of avian Plasmodium DNA in mosquito's head and thorax, where the salivary glands are located, provides valuable information on interactions between parasites and potential vectors. However, this does not fully guarantee the vector competence for avian malaria since DNA amplification could come from abortive stages of the parasite (Valkiunas, 2011; Valkiunas et al., 2013). To determine the transmission capacity of avian Plasmodium by the mosquito, visualization of sporozoites in the salivary glands or the extraction of saliva from the mosquito are more reliable methods (Valkiunas, 2005; Gutiérrez-López et al., 2016; Palinauskas et al., 2016). In order to dissect the salivary glands or obtain a saliva sample, the mosquito must be fresh or alive, and must not have been previously frozen. However, mosquito dissection is not always compatible with field conditions and the number of engorged mosquitoes that fed on infected birds that show parasites in their salivary glands or saliva is usually very low (between 1 and 5\%; Alves, 2012; Gutiérrez-López et al., 2020). In addition to fieldwork, experimental studies in laboratory conditions seem therefore essential to evaluate the competent mosquito species for avian Plasmodium lineages, even if it requires complex logistics to (i) test several lineages at the same time, and (ii) keep mosquitoes in colonies and birds in captivity. Among many lines of research that could improve our understanding of vector specificity, future projects should focus on (i) questioning the parasite 
transmission capacity of mammophilic mosquitoes since, so far, Plasmodium lineages have been found in head/thorax only and not in salivary glands, (ii) testing the vector competence of mosquitoes that share the same habitat as $C x$. pipiens but are present in lower abundance, (iii) determining if the infection status of the mosquitoes influences their host selection, using a wide range of mammals and bird species, or, (iv) evaluating if lineages with different host specialization strategies (specialists vs. generalists) are transmitted equally by mosquitoes.

\section{CONCLUSION}

Research projects based on a global approach to host-parasite relationships that integrates the pivotal role of vectors in avian malaria transmission are still very rare, but essential to understand the evolutionary strategies of parasites. Although molecular ecology tools helped make great strides, there is still a long way to go to understand parasite-vector-host relationships, especially given the high diversity of known parasites and potential vectors. To date, data from the literature suggest that avian Plasmodium lineages present a gradient of host specificity from highly specialist to highly generalist, and relatively high vector specificity (i.e., each lineage uses only a few vectors). However, true vector specificity of avian Plasmodium needs to be clarified by obtaining larger datasets about vector-parasite interactions in the field and by demonstrating vector competence. Among factors that could influence interactions between vectors and their hosts and parasites, the feeding behavior of mosquitoes may be a key factor that influences vector specialization, although this remains to be proven. Multidisciplinary teams, including ecologists, ornithologists, parasitologists and entomologists, should work hand in hand to understand fully the range of parasite evolutionary strategies in both hosts and vectors.

\section{REFERENCES}

Alcaide, M., Rico, C., Ruiz, S., Soriguer, R., MuÃtoz, J., and Figuerola, J. (2009). Disentangling vector-borne transmission networks: a universal DNA barcoding method to identify vertebrate hosts from arthropod bloodmeals. PLoS ONE 4:e7092. doi: 10.1371/journal.pone.0007092

Allan, S. A., Bernier, U. R., and Kline, D. L. (2006). Attraction of mosquitoes to volatiles associated with blood. J. Vector Ecol. 31, 71-78. doi: 10.3376/1081-171020063171:AOMTVA2.0.CO;2

Alves, R. O. (2012). Avian malaria associations with British mosquitoes (Dissertation/ PhD thesis). Oxford: University of Oxford.

Amo, L., Avilés, J. M., Parejo, D., Peña, A., Rodríguez, J., and Tomás, G. (2012). Sex recognition by odour and variation in the uropygial gland secretion in starlings. J. Anim. Ecol. 81, 605-613. doi: 10.1111/j.1365-2656.2011.01940.x

Aruch, S., Atkinson, C. T., Savage, A. F., and LaPointe, D. A. (2007). Prevalence and distribution of pox-like lesions, avian malaria, and mosquito vectors in Kipahulu valley, Haleakalā National Park, Hawai'i, USA. J. Wildl Dis. 43, 567-575. doi: 10.7589/0090-3558-43.4.567

Beadell, J. S., Covas, R., Gebhard, C., Beadell, J. S., Covas, R., Gebhard, C., et al. (2009). Host associations and evolutionary relationships of avian blood parasites from West Africa. Int. J. Parasitol. 39, 257-266. doi: 10.1016/j.ijpara.2008.06.005

Bennett, G. F. (1961). On the specificity and transmission of same avian Trypanosomes. Can. J. Zool. 39, 17-33. doi: 10.1139/z61-003

\section{DATA AVAILABILITY STATEMENT}

The raw data of this article will be made available by the authors, without undue reservation, to any qualified researcher.

\section{AUTHOR CONTRIBUTIONS}

RG-L and CL conceived the idea for this study, extracted the data from MalAvi, and wrote the first draft. CL carried out the statistical analyses. VB commented on the setup and assisted during the writing of the paper and revisions. All authors contributed to the article and approved the submitted version.

\section{FUNDING}

This work was funded by National Funds through FCT-Foundation for Science and Technology under the IF/00744/2014/CP1256/CT0001 Exploratory Research Project (CL) and the PTDC/BIA-EVL/29390/2017 DEEP Research Project (CL, RG-L, and VB).

\section{ACKNOWLEDGMENTS}

We sincerely thank Ravinder Sehgal for his suggestions and proofreading of the manuscript. We would also like to thank the three reviewers for their constructive comments on the manuscript.

\section{SUPPLEMENTARY MATERIAL}

The Supplementary Material for this article can be found online at: https://www.frontiersin.org/articles/10.3389/fevo. 2020.569230/full\#supplementary-material

Bensch, S., Hellgren, O., and Perez-Tris, J. (2009). MalAvi: a public database of malaria parasites and related haemosporidians in avian hosts based on mitochondrial cytochrome b lineages. Mol. Ecol. Res. 9, 1353-1358. doi: 10.1111/j.1755-0998.2009.02692.x

Bichet, C., Sorci, G., Robert, A., Julliard, R., Lendvai, A. Z., Chastel, O., et al. (2014). Epidemiology of Plasmodium relictum infection in the house sparrow. J. Parasitol. 100, 59-65. doi: 10.1645/12-24.1

Billingsley, P. F., and Sinden, R. E. (1997). Determinants of malaria-mosquito specificity. Parasitol. Today 13, 297-301. doi: 10.1016/S0169-4758(97)01094-6

Burkett-Cadena, N. D., Bingham, A. M., and Unnasch, T. R. (2014). Sexbiased avian host use by arbovirus vectors. R. Soc. Open. Sci. 1:140262. doi: 10.1098/rsos.140262

Burkett-Cadena, N. D., Graham, S. P., Hassan, H. K., Guyer, C., Eubanks, M. D., Katholi, C. R., et al. (2008). Blood feeding patterns of potential arbovirus vectors of the genus Culex targeting ectothermic hosts. Am. J. Trop. Med. Hyg. 79, 809-815. doi: 10.4269/ajtmh.2008.79.809

Calero-Riestra, M., and Garcia, J. T. (2016). Sex-dependent differences in avian malaria prevalence and consequences of infections on nestling growth and adult condition in the Tawny pipit, Anthus campestris. Malar J. 15:178. doi: 10.1186/s12936-016-1220-y

Carlson, J. S., Nelms, B., Barker, C. M., Reisen, W. K., Sehgal, R. N., and Cornel, A. J. (2018). Avian malaria co-infections confound infectivity and vector competence assays of Plasmodium homopolare. Parasitol. Res. 117, 2385-2394. doi: 10.1007/s00436-018-5924-5 
CDC (Centers for Disease Control and Prevention) (2019). West Nile Virus: Statistics, Surveillance and Control. Available online at: https://www.cdc.gov/ westnile/statsmaps/cumMapsData.html (accessed May 18, 2020).

Combes, C. (1997). Fitness of parasites: pathology and selection. Int. J. Parasitol. 27, 1-10. doi: 10.1016/S0020-7519(96)00168-3

Cornet, S., Nicot, A., Rivero, A., and Gandon, S. (2013). Malaria infection increases bird attractiveness to uninfected mosquitoes. Ecol. Lett. 16, 323-329. doi: 10.1111/ele.12041

Cozzarolo, C. S., Sironi, N., Glaizot, O., Pigeault, R., and Christe, P. (2019). Sex-biased parasitism in vector-borne disease: Vector preference? PLoS ONE 14:e0216360. doi: 10.1371/journal.pone.0216360

Díez-Fernández, A., Martínez-de la Puente, J., Gangoso, L., Ferraguti, M., Soriguer, R., and Figuerola, J. (2019). House sparrow uropygial gland secretions do not attract ornithophilic nor mammophilic mosquitoes. Med. Vet. Entomol. 34, 225-228. doi: 10.1111/mve.12401

Díez-Fernández, A., Martínez-de la Puente, J., Gangoso, L., López, P., Soriguer, R., Martín, J., et al. (2020). Mosquitoes are attracted by the odour of Plasmodium infected birds. Int. J. Parasitol. 50, 569-575. doi: 10.1016/j.ijpara.2020.03.013

Dobson, A. (2004). Population dynamics of pathogens with multiple host species. Am. Nat. 164, S64-S78. doi: 10.1086/424681

Dye, C., and Hasibeder, G. (1986). Population-dynamics of mosquito-borne disease: effects of flies which bite some people more frequently than others. Trans. R. Soc. Trop. Med. Hyg. 80, 69-77. doi: 10.1016/0035-9203(86) 90199-9

Eiras, A. E., and Jepson, P. C. (1994). Responses of female Aedes aegypti (Diptera: Culicidae) to host odours and convection currents using an olfactometer bioassay. B Entomol. Res. 84, 207-211. doi: 10.1017/S0007485300039705

Ejiri, H., Sato, Y., Kim, K. S., Tsuda, Y., Murata, K., Saito, K., et al. (2011). Blood meal identification and prevalence of avian malaria parasite in mosquitoes collected at Kushiro Wetland, a subarctic zone of Japan. J. Med. Entomol. 48, 904-908. doi: 10.1603/ME11053

Farajollahi, A., Fonseca, D. M., Kramer, L. D., and Kilpatrick, A. M. (2011). "Bird biting" mosquitoes and human disease: a review of the role of Culex pipiens complex mosquitoes in epidemiology. Infect. Genet. Evol. 11, 1577-1585. doi: 10.1016/j.meegid.2011.08.013

Fecchio, A., Wells, K., Bell, J. A., Tkach, V. V., Lutz, H. L., Weckstein, J. D., et al. (2019). Climate variation influences host specificity in avian malaria parasites. Ecol. Lett. 22, 547-557. doi: 10.1111/ele.13215

Ferraguti, M., Martínez-de la Puente, J., Muñoz, J., Roiz, D., Ruiz, S., Soriguer, R., et al. (2013). Avian Plasmodium in Culex and Ochlerotatus mosquitoes from southern Spain: effects of season and host-feeding source on parasite dynamics. PLoS ONE 8:e66237. doi: 10.1371/journal.pone.0066237

Ferraguti, M., Martínez-de La Puente, J., Roiz, D., Ruiz, S., Soriguer, R., and Figuerola, J. (2016). Effects of landscape anthropization on mosquito community composition and abundance. Sci. Rep. 6, 1-9. doi: $10.1038 /$ srep 29002

Gager, A. B., Del Rosario Loaiza, J., Dearborn, D. C., and Bermingham, E. (2008). Do mosquitoes filter the access of Plasmodium cytochrome b lineages to an avian host?. Mol. Ecol. 17, 2552-2561. doi: 10.1111/j.1365-294X.2008.03764.x

Glaizot, O., Fumagalli, L., Iritano, K., Lalubin, F., Van Rooyen, J., and Christe, P. (2012). High prevalence and lineage diversity of avian malaria in wild populations of Great tits (Parus major) and mosquitoes (Culex pipiens). PLoS ONE. 7:e34964. doi: 10.1371/journal.pone.0034964

Gomez, J. M., Verdu, M., and Perfectti, F. (2010). Ecological interactions are evolutionarily conserved across the entire tree of life. Nature 465, 918-921. doi: 10.1038/nature09113

Gutiérrez-López, R., Martínez-de la Puente, J., Gangoso, L., Soriguer, R., and Figuerola, J. (2019). Effects of host sex, body mass and infection by avian Plasmodium on the biting rate of two mosquito species with different feeding preferences. Parasite Vector 12:87. doi: 10.1186/s13071-019-3342-x

Gutiérrez-López, R., Martínez-de la Puente, J., Gangoso, L., Soriguer, R., and Figuerola, J. (2020). Plasmodium transmission differs between mosquito species and parasite lineages. Parasitology 147, 441-447. doi: $10.1017 /$ S0031182020000062

Gutiérrez-López, R., Martínez-de la Puente, J., Gangoso, L., Yan, J., Soriguer, R. C., and Figuerola, J. et al. (2016). Do mosquitoes transmit the avian malarialike parasite Haemoproteus? An experimental test of vector competence using mosquito saliva. Parasite Vector 9:609. doi: 10.1186/s13071-016-1903-9
Hamer, G. L., Kitron, U. D., Brawn, J. F., Loss, S. R., Ruiz, M. O., Goldberg, T. L., et al. (2008). Culex pipiens (Diptera: Culicidae): a bridge vector of West Nile virus to humans. J. Med. Entomol. 45, 125-128. doi: 10.1093/jmedent/45.1.125

Hamer, G. L., Kitron, U. D., Goldberg, T. L., Brawn, J. D., Loss, S. R., Ruiz, M. O., et al. (2009). Host selection by Culex pipiens mosquitoes and West Nile virus amplification. Am. J. Trop. Med. Hyg. 80, 268-278. doi: 10.4269/ajtmh.2009.80.268

Heil, M. (2016). Host manipulation by parasites: cases, patterns, and remaining doubts. Front. Ecol. Evol. 4:80. doi: 10.3389/fevo.2016.00080

Hellgren, O., Bensch, S., and Malmqvist, B. (2008). Bird hosts, blood parasites and their vectors - associations uncovered by molecular analyses of blackfly blood meals. Mol. Ecol. 17, 1605-1613. doi: 10.1111/j.1365-294X.2007.03680.x

Hellgren, O., Pérez-Tris, J., and Bensch, S. (2009), A jack-of-all-trades and still a master of some: prevalence and host range in avian malaria and related blood parasites. Ecology 90, 2840-2849. doi: 10.1890/08-1059.1

Hurd, H. (2003). Manipulation of medically important insect vectors by their parasites. Annu. Rev. Entomol. 48, 141-161. doi: 10.1146/annurev.ento.48.091801.112722

Inci, A., Yildirim, A., Njabo, K. Y., Duzlu, O., Biskin, Z., and Ciloglu, A. (2012). Detection and molecular characterization of avian Plasmodium from mosquitoes in central Turkey. Vet. Parasitol. 188, 179-184. doi: 10.1016/j.vetpar.2012.02.012

Ishtiaq, F., Guillaumot, L., Clegg, S. M., Phillimore, A. B., Black, R. A., Owens, I. P. F., et al. (2008). Avian haematozoan parasites and their associations with mosquitoes across Southwest Pacific Islands. Mol. Ecol. 17, 4545-4555. doi: 10.1111/j.1365-294X.2008.03935.x

Jacob, J., Balthazart, J., and Schoffeniels, E. (1979). Sex differences in the chemical composition of uropygial gland waxes in domestic ducks. Biochem. Syst. Ecol. 7, 149-153. doi: 10.1016/0305-1978(79)90024-3

Johnson, K. P., Malenke, J. R., and Clayton, D. H. (2009). Competition promotes the evolution of host generalists in obligate parasites. Proc. Biol. Sci. 276, 3921-3926. doi: 10.1098/rspb.2009.1174

Kamgang, B., Nchoutpouen, E., Simard, F., and Paupy, C. (2012). Notes on the blood-feeding behavior of Aedes albopictus (Diptera: Culicidae) in Cameroon. Parasite Vector 5:57. doi: 10.1186/1756-3305-5-57

Kazlauskiene, R., Bernotiene, R., Palinauskas, V., Iezhova, T. A., and Valkiunas, G. (2013). Plasmodium relictum (lineages pSGS1 and pGRW11): complete synchronous sporogony in mosquitoes Culex pipiens pipiens. Exp. Parasitol. 133, 454-461. doi: 10.1016/j.exppara.2013.01.008

Kelley, S. T., and Farrell, B. D. (1998). Is specialization a dead end? The phylogeny of host use in Dendroctonus bark beetles (Scolytidae). Evolution 52, 1731-1743. doi: 10.1111/j.1558-5646.1998.tb02253.x

Kilpatrick, A. M., Kramer, L. D., Jones, M. J., Marra, P. P., and Daszak, P. (2006). West Nile virus epidemics in North America are driven by shifts in mosquito feeding behaviour. PLoS. Biol. 4:e82. doi: 10.1371/journal.pbio.0040082

Kimura, M., Darbro, J. M., and Harrington, L. C. (2010). Avian malaria parasites share congeneric mosquito vectors. J. Parasitol. 96, 144-151. doi: 10.1645/GE-2060.1

Kleiber, M. (1947). Body size and metabolic rate. Physiol. Rev. 27, 511.541. doi: 10.1152/physrev.1947.27.4.511

Lalubin, F., Bize, P., van Rooyen, J., Christe, P., and Glaizot, O. (2012). Potential evidence of parasite avoidance in an avian malarial vector. Anim. Behav. 84, 539-545. doi: 10.1016/j.anbehav.2012.06.004

Lalubin, F., Delédevant, A., Glaizot, O., and Christe, P. (2013). Temporal changes in mosquito abundance (Culex pipiens), avian malaria prevalence and lineage composition. Parasite Vector 6:307. doi: 10.1186/1756-3305-6-307

LaPointe, D. A., Atkinson, C. T., and Samuel, M. D. (2012). Ecology and conservation biology of avian malaria. Ann. N. Y. Acad. Sci. 1249, 211-226. doi: 10.1111/j.1749-6632.2011.06431.x

Lauron, E., Loiseau, C., Bowie, R., Spicer, G., Smith, T., Melo, M., et al. (2015). Coevolutionary patterns and diversification of avian malaria parasites in African sunbirds (Family Nectariniidae). Parasitology 142, 635-647. doi: $10.1017 /$ S0031182014001681

Lefévre, T., and Thomas, F. (2008). Behind the scene, something else is pulling the strings: emphasizing parasitic manipulation in vector-borne diseases. Infect. Genet. Evol. 8, 504-519. doi: 10.1016/j.meegid.2007.05.008

Lehane, M. J. (2005). The Biology of Blood-Sucking Insects. Cambridge: Cambridge University Press. 
Liebman, K. A., Stoddard, S. T., Reiner, R. C. Jr., Perkins, T. A., Astete, H., Sihuincha, M., et al. (2014). Determinants of heterogeneous blood feeding patterns by Aedes aegypti in Iquitos, Peru. PLoS Neglect. Trop. Dis. 8:e2702. doi: 10.1371/journal.pntd.0002702

Loiseau, C., Harrigan, R. J., Robert, A., Bowie, R. C. K., Thomassen, H. A., Smith, T. B., et al. (2012). Host and habitat specialization of avian malaria in Africa. Mol. Ecol. 21, 431-441. doi: 10.1111/j.1365-294X.2011.05341.x

Loiseau, C., Zoorob, R., Garnier, S., Birard, J., Federici, P., Julliard, R., et al. (2008). Antagonistic effects of a $M h c$ class I allele on malaria-infected house sparrows. Ecol. Lett. 11, 258-265. doi: 10.1111/j.1461-0248.2007.01141.x

Lura, T., Cummings, R., Velten, R., De Collibus, K., Morgan, T., Nguyen, K., et al. (2012). Host (avian) biting preference of southern California Culex mosquitoes (Diptera: Culicidae). J. Med. Entomol. 49, 687-696. doi: 10.1603/ME11177

Marquardt, W. C., Black, W. C., Freier, J. E., Hagedorn, H. H., Hemingway, J., Higgs, S., et al. (2005). Biology of Disease Vectors. Burlington, MA: Elsevier.

Martínez-de la Puente, J., Díez-Fernández, A., Montalvo, T., Bueno-Mar,í, R., Pangrani, Q., Soriguer, R. C., et al. (2020). Do invasive mosquito and bird species alter avian malaria parasite transmission? Diversity 12:111. doi: $10.3390 / \mathrm{d} 12030111$

Martínez-de la Puente, J., Ferraguti, M., Ruiz, S., Roiz, D., Soriguer, R. C., and Figuerola, J. (2016). Culex pipiens forms and urbanization: effects on blood feeding sources and transmission of avian Plasmodium. Malar. J. 15:589. doi: 10.1186/s12936-016-1643-5

Martínez-de la Puente, J., Martinez, J., Rivero-de Aguilar, J., Herrero, J., and Merino, S. (2011). On the specificity of avian blood parasites: revealing specific and generalist relationships between haemosporidians and biting midges. Mol. Ecol. 20, 3275-3287. doi: 10.1111/j.1365-294X.2011.05136.x

Martínez-de la Puente, J., Merino, S., Lobato, E., Rivero-de Aguilar, J., del Cerro, S., Ruiz-de-Castañeda, R., et al. (2010). Nest-climatic factors affect the abundance of biting flies and their effects on nestling condition. Acta Oecol. 36, 543-547. doi: 10.1016/j.actao.2010.07.008

Martínez-de la Puente, J., Muñoz, J., Capelli, G., Montarsi, F., Soriguer, R., Arnoldi, D., et al. (2015). Avian malaria parasites in the last supper: identifying encounters between parasites and the invasive Asian mosquito tiger and native mosquito species in Italy. Malar J. 14:32. doi: 10.1186/s12936-015-0571-0

Mayi, M. P. A., Bamou, R., Djiappi-Tchamen, B., Fontaine, A., Jeffries, C. L., Walker, T., et al. (2020). Habitat and seasonality affect mosquito community composition in the west region of Cameroon. Insects 11:312. doi: $10.3390 /$ insects 11050312

Mccoy, K., Léger, E., and Dietrich, M. (2013). Host specialization in ticks and transmission of tick-borne diseases: a review. Front. Cell. Infect. Microbiol. 3:57. doi: 10.3389/fcimb.2013.00057

Molaei, G., Andreadis, T. G., Armstrong, P. M., and Diuk-Wasser, M. (2007). Host-feeding patterns of potential mosquito vectors in Connecticut, USA: molecular analysis of bloodmeals from 23 species of Aedes, Anopheles, Culex, Coquillettidia, Psorophora, and Uranotaenia. J. Med. Entomol. 45, 1143-1151. doi: 10.1093/jmedent/45.6.1143

Muñoz, J., Ruiz, S., Soriguer, R., Alcaide, M., Viana, D. S., Roiz, D., et al. (2012). Feeding patterns of potential West Nile virus vectors in south-west Spain. PLoS ONE 7:e39549. doi: 10.1371/journal.pone.0039549

Njabo, K. Y., Cornel, A. J., Bonneaud, C., Toffelmier, E., Sehgal, R. N. M., Valkiunas, G., et al. (2011). Nonspecific patterns of vector, host and avian malaria parasite associations in a central African rainforest. Mol. Ecol. 20, 1049-1061. doi: 10.1111/j.1365-294X.2010.04904.x

Njabo, K. Y., Cornel, A. J., Sehgal, R. N., Loiseau, C., Buermann, W., Harrigan, R. J., et al. (2009). Coquillettidia (Culicidae, Diptera) mosquitoes are natural vectors of avian malaria in Africa. Malar. J. 8:193. doi: 10.1186/1475-2875-8-193

Nosil, P. (2002). Transition rates between specialization and generalization in phytophagous insects. Evolution 56, 1701-1706. doi: 10.1111/j.0014-3820.2002.tb01482.x

Palinauskas, V., Žiegyte, R., Iezhova, T. A., Ilgunas, M., Bernotien,e, R., and Valkiunas, G. (2016). Description, molecular characterisation, diagnostics and life cycle of Plasmodium elongatum (lineage pERIRUB01), the virulent avian malaria parasite. Int. J. Parasitol. 46, 697-707. doi: 10.1016/j.ijpara.2016.05.005

Panella, N. A., Crockett, R. J., Biggerstaff, B. J., and Komar, N. (2011). The Centers for Disease Control and Prevention resting trap: a novel device for collecting resting mosquitoes. J. Am. Mosq. Control. Assoc. 27, 323-325. doi: 10.2987/09-5900.1
Perkins, T. A., Scott, T. W., Le Menach, A., and Smith, D. L. (2013). Heterogeneity, mixing, and the spatial scales of mosquito-borne pathogen transmission. PLoS Comput. Biol. 9:e1003327. doi: 10.1371/journal.pcbi.1003327

Poisot, T., Kéfi, S., Morand, S., Stanko, M., Marquet, P. A., and Hochberg, M. E. (2015). A continuum of specialists and generalists in empirical communities. PLoS ONE 10:e0114674. doi: 10.1371/journal.pone.0114674

Poulin, R. (2000). Manipulation of host behaviour by parasites: a weakening paradigm? Proc. Biol. Sci. 267, 787-792. doi: 10.1098/rspb.2000.1072

Poulin, R. (2007). Evolutionary Ecology of Parasites. Princeton, NJ: Princeton University Press.

Poulin, R., Krasnov, B. R., Shenbrot, G. I., Mouillot, D., and Khoklova, S. (2006) Evolution of host specificity in fleas: is it directional and irreversible? Int. J. Parasitol. 36, 185-191. doi: 10.1016/j.ijpara.2005.09.017

Raji, J. I., and DeGennaro, M. (2017). Genetic analysis of mosquito detection of humans. Curr. Opin. Insect. Sci. 20, 34-38. doi: 10.1016/j.cois.2017.03.003

Ricklefs, R. E., and Fallon, S. M. (2002). Diversification and host switching in avian malaria parasites. Proc. Biol. Sci. 269, 885-892. doi: 10.1098/rspb.2001.1940

Ricklefs, R. E., Fallon, S. M., and Bermingham, E. (2004). Evolutionary relationships, cospeciation, and host switching in avian malaria parasites. Syst. Biol. 53, 111-119. doi: 10.1080/10635150490264987

Ricklefs, R. E., Outlaw, D. C., Svensson-Coelho, M., Medeiros, M. C. I., Ellis, V. A., et al. (2014). Species formation by host shifting in avian malaria parasites. Proc. Natl. Acad. Sci. U.S.A. 111, 14816-14821. doi: 10.1073/pnas.1416356111

Rivero, A., and Gandon, S. (2018). Evolutionary ecology of avian malaria: past to present. Trends. Parasitol. 34, 712-726. doi: 10.1016/j.pt.2018.06.002

Rizzoli, A., Bolzoni, L., Chadwick, E. A., Capelli, G., Montarsi, F., Grisenti, M., et al. (2015). Understanding West Nile virus ecology in Europe: Culex pipiens host feeding preference in a hotspot of virus emergence. Parasite Vector 8:213. doi: 10.1186/s13071-015-0831-4

Russell, C. B., and Hunter, F. F. (2005). Attraction of culex pipiens/restuans (Diptera: Culicidae) mosquitoes to bird uropygial gland odors at two elevations in the Niagara region of Ontario. J. Med. Entomol. 42, 301-305. doi: 10.1093/jmedent/42.3.301

Santiago-Alarcon, D., Palinauskas, V., and Schaefer, H. M. (2012). Diptera vectors of avian Haemosporidian parasites: untangling parasite life cycles and their taxonomy. Biol. Rev. 87, 928-964. doi: 10.1111/j.1469-185X.2012.00234.x

Savage, H. M., Aggarwal, D., Apperson, C. S., Katholi, C. R., Gordon, E., Hassan, H. K., et al. (2007). Host choice and West Nile virus infection rates in blood-fed mosquitoes, including members of the Culex pipiens complex, from Memphis and Shelby County, Tennessee, 2002-2003. Vector Borne Zoonotic Dis. 7, 365-386. doi: 10.1089/vbz.2006.0602

Schönenberger, A. C., Wagner, S., Tuten, H. C., Schaffner, F., Torgerson, P., Furrer, S., et al. (2016). Host preferences in host-seeking and blood-fed mosquitoes in Switzerland. Med. Vet. Entomol. 30, 39-52. doi: 10.1111/mve.12155

Simpson, J. E., Folsom-O’Keefe, C. M., Childs, J. E., Simons, L. E., Andreadis, T. G., and Diuk-Wasser, M. A. (2009). Avian host-selection by Culex pipiens in experimental trials. PLoS ONE 4:e7861. doi: 10.1371/journal.pone.00 07861

Skorping, A., and Jensen, K. H. (2004). Disease dynamics: all caused by males? Trends Ecol. Evol. 19, 219-220. doi: 10.1016/j.tree.2004.02.006

Snyder, S. D., and Loker, E. S. (2000). Evolutionary relationships among the Schistosomatidae (Platyhelminthes: Digenea) and an Asian origin for Schistosoma. J. Parasitol. 86, 283-288. doi: 10.1645/0022-339520000860283:ERATSP2.0.CO

Svobodová, M., Dolnik, O. V., Cepička, I., and Rádrová, J. (2017). Biting midges (Ceratopogonidae) as vectors of avian trypanosomes. Parasite Vector 10:224. doi: 10.1186/s13071-017-2158-9

Takken, W., and Verhulst, N. O. (2013). Host preferences of blood-feeding mosquitoes. Annu. Rev. Entomol. 58, 433-453. doi: 10.1146/annurev-ento-120811-153618

Thiemann, T. C., Wheeler, S. S., Barker, C. M., and Reisen, W. K. (2011). Mosquito host selection varies seasonally with host availability and mosquito density. PLoS Negl. Trop. Dis. 5:e1452. doi: 10.1371/journal.pntd.0001452

Valkiunas, G. (2005). Avian Malaria Parasites and Other Haemosporidia. Boca Raton, FL: CRC Press.

Valkiunas, G. (2011). Haemosporidian vector research: marriage of molecular and microscopical approaches is essential. Mol. Ecol, 20, 3084-3086. doi: 10.1111/j.1365-294X.2011.05187.x 
Valkiunas, G., Kazlauskiene, R., Bernotiene, R., Palinauskas, V., and JeŽova, T. (2013). Abortive long-lasting sporogony of two Haemoproteus species (Haemosporida, Haemoproteidae) in the mosquito Ochlerotatus cantans, with perspectives on haemosporidian vector research. Parasitol. Res. 112, 2159-2169. doi: 10.1007/s00436-013-3375-6

Vázquez, D. P., Poulin, R., Krasnov, B. R., and Shenbrot, G. I. (2005). Species abundance and the distribution of specialization in host-parasite interaction networks. J. Anim. Ecol. 74, 946-955. doi: 10.1111/j.1365-2656.2005.00992.x

Viana, L. A., Soares, P., Paiva, F., and Lourenço-de-Oliveira, R. (2010). CaimanBiting mosquitoes and the natural vectors of Hepatozoon caimani in Brazil. J. Med. Entomol. 47, 670-676. doi: 10.1093/jmedent/7.4.670

Wekesa, J. W., Yuval, B., Washino, R. K., and de Vasquez, A. M. (1997). Blood feeding patterns of Anopheles freeborni and Culex tarsalis (Diptera: Culicidae): effects of habitat and host abundance. B. Entomol. Res. 87, 633-641. doi: 10.1017/S0007485300038736

Wilson, A. J., Morgan, E. R., Booth, M., Norman, R., Perkins, S. E., Hauffe, H. C., et al. (2017). What is a vector? Phil. Trans. R. Soc. B. 372:20160085. doi: $10.1098 /$ rstb.2016.0085

Woolhouse, M. E. J., Dye, C., Etard, J.-F., Smith, T., Charlwood, J. D., Garnett, G. P., et al. (1997). Heterogeneities in the transmission of infectious agents: implications for the design of control programs. Proc. Natl. Acad. Sci. U.S.A. 94, 338-342. doi: 10.1073/pnas.94.1.338

Yan, J., Gangoso, L., Martínez-de la Puente, J., Soriguer, R., and Figuerola, J. (2017). Avian phenotypic traits related to feeding preferences in two Culex mosquitoes. Sci. Nat. Heidelberg 104:76. doi: 10.1007/s00114-017-1497-x
Yan, J., Martínez-de la Puente, J., Gangoso, L., Gutiérrez-López, R., Soriguer, R., and Figuerola, J. (2018). Avian malaria infection intensity influences mosquito feeding patterns. Int. J. Parasitol. 48, 257-264. doi: 10.1016/j.ijpara.2017. 09.005

Zélé, F., Vézilier, J., L’ambert, G., Nicot, A., Gandon, S., Rivero, A., et al. (2014). Dynamics of prevalence and diversity of avian malaria infections in wild Culex pipiens mosquitoes: the effects of Wolbachia, filarial nematodes and insecticide resistance. Parasite Vector 7:437. doi: 10.1186/1756-3305-7-437

Zuk, M., and Stoehr, A. M. (2010). "Sex differences in susceptibility to infection: an evolutionary perspective," in: Sex Hormones and Immunity to Infection, eds S. L. Klein and C. W. Robert (Berlin: Springer), 1-17.

Zuk, M., Thornhill, R., Ligon, J. D., and Johnson, K. (1990). Parasites and mate choice in red jungle fowl. Am. Zool. 30, 235-244. doi: 10.1093/icb/30.2.235

Conflict of Interest: The authors declare that the research was conducted in the absence of any commercial or financial relationships that could be construed as a potential conflict of interest.

Copyright (c) 2020 Gutiérrez-López, Bourret and Loiseau. This is an open-access article distributed under the terms of the Creative Commons Attribution License (CC $B Y)$. The use, distribution or reproduction in other forums is permitted, provided the original author(s) and the copyright owner(s) are credited and that the original publication in this journal is cited, in accordance with accepted academic practice. No use, distribution or reproduction is permitted which does not comply with these terms. 